\title{
AN EFFECT OF NERVE GROWTH FACTOR ON THE PARASYMPATHETIC CILIARY GANGLION ${ }^{1}$
}

\author{
FRANK COLLINS
}

Department of Anatomy, University of Utah School of Medicine, Salt Lake City, Utah 84132

Received August 15, 1983; Revised November 23, 1983; Accepted November 23, 1983

\begin{abstract}
Mouse submaxillary nerve growth factor (NGF) increases the length and density of neurites extended from embryonic chick ciliary ganglia in vitro. The effect is not as pronounced as with dorsal root ganglia, but it occurs at the same low concentrations of NGF. This effect was observed on polyornithine substrata with or without pretreatment of the substratum with conditioned medium. NGF did not significantly increase the length or density of neurites extended from spinal cord or neural retina explants in the same culture conditions. Stimulation of ciliary ganglion neurite outgrowth did not occur when NGF was replaced with cytochrome $c$ or epidermal growth factor, whereas insulin had an effect comparable to that of NGF when present at much higher concentrations. Four different purified preparations of NGF gave identical results. The purity of two of these preparations was determined after radioiodination; greater than $98 \%$ of the radioactivity migrated as a single band on polyacrylamide gels, and greater than $94 \%$ of the radioactivity was bound by affinity purified anti-NGF antibody. The effect of NGF on neurite growth could be separated from its effect on neuronal survival. NGF failed to promote the survival of ciliary ganglion neurites even at concentrations 1000 -fold higher than those which significantly stimulated neurite growth. The present results extend the growing body of recent evidence that specific populations of central and peripheral cholinergic neurons can respond to NGF.
\end{abstract}

Nerve growth factor (NGF) is a polypeptide hormone that plays an important role in development and function of peripheral sympathetic and sensory neurons (Greene and Shooter, 1980; Harper and Thoenen, 1980). NGF promotes fiber outgrowth, survival, and the synthesis of key transmitter-related enzymes in these neurons. Recent evidence indicates that specific populations of cholinergic neurons in the peripheral (Collins and Dawson, 1983) and central (Honegger and Lenoir, 1982; Gnahn et al., 1983) nervous systems also respond to NGF, with increased levels of choline acetyltransferase or with enhanced fiber outgrowth. These neurons also exhibit specific uptake and retrograde axonal transport of NGF in vivo (Max et al., 1978; Schwab et al., 1979). It seems worthwhile to study the effect of NGF on these cholinergic neurons in more detail in order to understand the possible role of NGF or NGF-related molecules in their normal development.

The present report extends our recent finding that physiological concentrations of NGF stimulate neurite outgrowth from isolated parasympathetic cholinergic

\footnotetext{
${ }^{1}$ The technical assistance of Margaret Vogel in these experiments is gratefully acknowledged. This work was supported by a grant from the National Science Foundation.
}

neurons of the chick ciliary ganglion (Collins and Dawson, 1983). The present experiments compare the effects of NGF on intact ciliary and dorsal root ganglia in terms of the degree of NGF stimulation of neurite outgrowth, the dose-response curves of both ganglia to NGF, and the effect of NGF on neuronal survival. Intact ciliary ganglia were used in the present experiments to exclude the possibility that the previously reported response of these neurons to NGF was an artifact of dissociation of the ganglion. The neuronal specificity of the response to NGF in our culture conditions was assessed using explants of embryonic spinal cord and neural retina. The molecular specificity of the effect of NGF was evaluated by comparison with the effects of proteins with analogous physical and chemical properties. Finally, the purity of some of the NGF preparations used was assessed by polyacrylamide gel electrophoresis and immunoprecipitation of radioiodinated material.

\section{Materials and Methods}

Culture conditions. Ciliary ganglia, dorsal root ganglia, the lumbar segment of the spinal cord, and neural retinas were taken from White Leghorn chicken embryos that had been incubated for 6 to 9 days. Ganglia were cleaned free of other tissues and cut in half; spinal cord and 
neural retinas were cut into pieces approximately equal in size to the ganglia. Explants were placed in $35-\mathrm{mm}$ plastic tissue culture dishes (Falcon) containing $1.5 \mathrm{ml}$ per dish of complete culture medium with varying amounts of test substances. Complete medium consisted of Ham's F12 (GIBCO) supplemented with $10 \%$ fetal calf serum (Irvine Scientific), 100 units per $\mathrm{ml}$ of penicillin, $100 \mu \mathrm{g}$ per $\mathrm{ml}$ of streptomycin, and $0.25 \mu \mathrm{g}$ per $\mathrm{ml}$ of fungizone (GIBCO).

Cultures dishes were coated with polyornithine before use (Collins, 1978a). In some experiments polyornithinecoated dishes were also subsequently exposed to the conditioned medium from a line of mouse parietal endoderm cells (Lehman et al., 1974). Such medium coats the substratum with a conditioning factor that promotes neurite outgrowth (Collins, 1978b).

Index of neurite growth (reticle assay). At 20 to $24 \mathrm{hr}$ after cultures were set up, the extent of the neurite network surrounding each explant was estimated using the following assay. One side of a square eyepiece reticle was aligned with one edge of the explant (chosen at random), and the number of neurites crossing the opposite side of the reticle was counted. This represented a distance of approximately $400 \mu \mathrm{m}$ from the edge of the explant. This process was repeated for four orthogonally directed sites for each explant, and at least six explants were counted per culture. 'Ihese results are reported in terms of the mean total number of neurites counted per explant in each culture. Where fasciculation occurred, the number of neurites in each bundle was estimated either by following it to the point where the neurites separated or by comparing its thickness to the thickness of single neurites. The neurites extended from pieces of neural retina were too fasciculated and not sufficiently long to be measured in this way. Therefore, for quantitation of neural retina outgrowth, the area of neuritic carpet around each explant was measured by counting the number of reticle squares containing neurites.

Index of neurite growth ( 0 to 4 scale). The long time required to perform the preceding quantitative assay, during which cultures were kept at room temperature, seemed to have an adverse effect on their subsequent survival. Therefore, for the experiments reported in Figures 3 and 4 , in which long-term cultures were required, a more rapid semiquantitative assay for neurite outgrowth was performed each day for several days on the same cultures. In this assay (see Collins and Lee, 1982 for details), the density of outgrowth around each of at least six explants per culture was estimated on a scale of 0 (few neurites) to 4 (densest outgrowth). The cultures were coded in such a way that the person scoring the outgrowth was unaware of their contents. Although the scores are consistent within each neuronal type, similar scores are not directly comparable between different types of ganglia.

Iodination. Iodination was carried out on $20 \mu \mathrm{g}$ of NGF in $70 \mu \mathrm{l}$ of $0.15 \mathrm{M}$ phosphate buffer, $\mathrm{pH} 7.2$, by sequential addition of $50 \mu \mathrm{l}$ of Enzymobead Reagent (Bio-Rad), 1.5 $\mathrm{mCi}$ of $\mathrm{Na}^{125} \mathrm{I}$ in $15 \mu \mathrm{l}$, and $25 \mu \mathrm{l}$ of $2 \% \alpha$-D-glucose (after mutarotation overnight). After $20 \mathrm{~min}$ at room temperature, the mixture was spun for $3 \mathrm{~min}$ in a microfuge to pellet the beads. An aliquot of the supernatant was removed to determine the specific activity by precipitation with trichloroacetic acid (TCA), and the remainder was loaded onto a 0.4 by $12 \mathrm{~cm}$ column of G-25 and eluted with Hank's balanced salt solution, buffered to pH 7.2 with HEPES. The first peak of radioactivity was collected as $\left[{ }^{124} \mathrm{I}\right]$ NGF. Greater than $98 \%$ of the radioactivity in this fraction was precipitated in $10 \%$ TCA using calf serum as carrier.

Polyacrylamide gel electrophoresis. Slab gels, containing a 10 to $25 \%$ linear gradient of polyacrylamide, were prepared according to the method of Studier (1973) and were used with the discontinuous buffer system of Laemmli (1970) containing $0.1 \%$ sodium dodecyl sulfate. A $5 \%$ polyacrylamide spacer gel was used on top of the separating gel. Samples were prepared in buffer containing $2 \%$ dodecyl sulfate and $2 \% 2$-mercaptoethanol and were heated to $95^{\circ} \mathrm{C}$ for $2 \mathrm{~min}$. Gels were fixed, then vacuum dried and used to expose x-ray film for autoradiography. Density tracings of the autoradiograms were made using a Gilford Multi Media Densitometer.

Materials. Purified mouse submaxillary gland $\beta-\mathrm{NGF}$ was the gift of Drs. A. Sutter and E. Shooter (Stanford University, Stanford, CA) and was also obtained from The Research Foundation of the State University of New York (Albany). Purified mouse submaxillary 2.5S NGF, prepared in the laboratory of Dr. W. Frazier (Washington University, St. Louis, MO), was the gift of Dr. Lester Partlow (University of Utah School of Medicine, Salt Lake City) and was also obtained from Collaborative Research. Mouse parietal endoderm cell line (PYS) was the gift of Dr. I. Lehman (University of Colorado Medical Center, Denver). Affinity purified rabbit anti-NGF antibody to mouse submaxillary $\beta$-NGF was obtained from LAREFsa (Switzerland). Receptor grade epidermal growth factor was obtained from KOR, Inc. (Cambridge, MA). Bovine pancreatic insulin was obtained from Sigma (St. Louis, MO).

\section{Results}

NGF caused an increase in the density and length of neurites extended from ciliary ganglia (Tables I and II, Figs. 1 and 2). These effects were readily apparent upon visual inspection of the cultures (Fig. 1) and were highly statistically significant when measured quantitatively (Tables I and II, Fig. 2). Similar responses were observed with all preparations of NGF tested (see "Materials and

\section{TABLE I}

Effect of NGF on ciliary and dorsal root ganglia

E.9 ciliary or dorsal root ganglia were cultured in complete medium with or without NGF (from Drs. Sutter and Shooter) on a polyornithine-coated substratum. After $24 \mathrm{hr}$, the neurite index (reticle assay) was determined for seven to eight explants per culture $(N)$. The value given is the mean and standard deviation for the combined data from duplicate cultures. The significance of the difference between control and NGF cultures ( $p$ value) was determined using the Mann-Whitney $U$ test on the combined data.

\begin{tabular}{|c|c|c|c|c|c|}
\hline \multirow{2}{*}{ Ganglia } & \multicolumn{2}{|c|}{ Without NGF } & \multicolumn{2}{|c|}{$\mathrm{NGF}(5 \mathrm{ng} / \mathrm{ml})$} & \multirow{2}{*}{$p$} \\
\hline & $\mathrm{N}$ & Neurite Index & $\mathrm{N}$ & Neurite Index & \\
\hline Ciliary & 16 & $1.2 \pm 1.8$ & 16 & $25.0 \pm 12.4$ & $<0.001$ \\
\hline Dorsal root & 15 & $17.6 \pm 7.8$ & 16 & $101.2 \pm 21.7$ & $<0.001$ \\
\hline
\end{tabular}


Methods") and were also observed whether or not the polyornithine substratum had been pretreated with conditioned medium. The results of Figures 1 and 4 and Table II were obtained with conditioned medium pretreatment of the substratum, whereas those of Figures 2, 3,5 and Table I were obtained without pretreatment.

There was considerable variation in the density of neurite outgrowth among individual ciliary ganglia in the same dish in both the control and the NGF-treated cultures. To illustrate this variation, Figure 1 includes

TABLE II

Effect of NGF on explants of spinal cord and neural retina

E.7 ciliary ganglia or explants of spinal cord or neural retina were cultured in complete medium with or without NGF (from Drs. Sutter and Shooter) on a polyornithine-coated substratum pretreated with conditioned medium. After $24 \mathrm{hr}$, the neurite index (reticle assay) was determined for the 8 to 12 explants per culture $(\mathrm{N})$. See "Materials and Methods" for the special assay used with explants of neural retina. The value given is the mean and standard deviation for the combined data from duplicate cultures. The significance of the difference between control and NGF cultures ( $p$ value) was determined using the MannWhitney $U$ test.

\begin{tabular}{|c|c|c|c|c|c|}
\hline \multirow{2}{*}{ Explant } & \multicolumn{2}{|c|}{ Without NGF } & \multicolumn{2}{|c|}{$10 \mathrm{ng} / \mathrm{ml}$ of $\mathrm{NGF}$} & \multirow{2}{*}{$p$} \\
\hline & $\mathrm{N}$ & Neurite Index & $\mathrm{N}$ & Neurite Index & \\
\hline Ciliary & 8 & $14.0 \pm 12.4$ & 8 & $88.1 \pm 22.6$ & $<0.001$ \\
\hline Spinal cord & 12 & $16.5 \pm 15.8$ & 12 & $17.9 \pm 12.7$ & $>0.1$ \\
\hline Neural retina & 12 & $37.3 \pm 7.5$ & 12 & $40.9 \pm 8.7$ & $>0.1$ \\
\hline
\end{tabular}

photographs of two ganglia from the control and two from the NGF-treated ciliary ganglion cultures to show the upper and lower range of observed outgrowth.

The response of ciliary ganglia to NGF was compared to the response of dorsal root ganglia, which has been well studied. The dose-response curves to NGF of dorsal root and ciliary ganglia were similar (Fig. 2). With both types of ganglia there was a significant increase in neurite growth at $0.1 \mathrm{ng} / \mathrm{ml}$ of NGF, reaching a maximum at 10 $\mathrm{ng} / \mathrm{ml}$ (Fig. 2). NGF caused a striking increase in the density of neurites extended from dorsal root ganglia (Table I, Fig. 1). In response to NGF, some ciliary ganglia achieved neurite densities comparable to those of the dorsal root ganglia; however, the average response of ciliary ganglia was more modest (Table I, Fig. 1). Ciliary ganglia also showed less outgrowth in the absence of NGF than did dorsal root ganglia (Table I, Fig. 1).

Occasionally, there was a decreased response to NGF at concentrations greater than $10 \mathrm{ng} / \mathrm{ml}$, which is not illustrated by the data in Figure 2 (however, see Collins and Dawson, 1983). On these occasions, concentrations between 1 and $10 \mathrm{ng} / \mathrm{ml}$ seemed to give the best response.

Survival. The stimulatory effects of NGF on neurite growth in dorsal root and ciliary ganglia were analogous, but the effects of NGF on neurite survival were strikingly different in the two types of ganglia (Fig. 3). NGF failed to promote survival of the neurites extended from ciliary ganglia, even at concentrations of NGF 1000 -fold higher

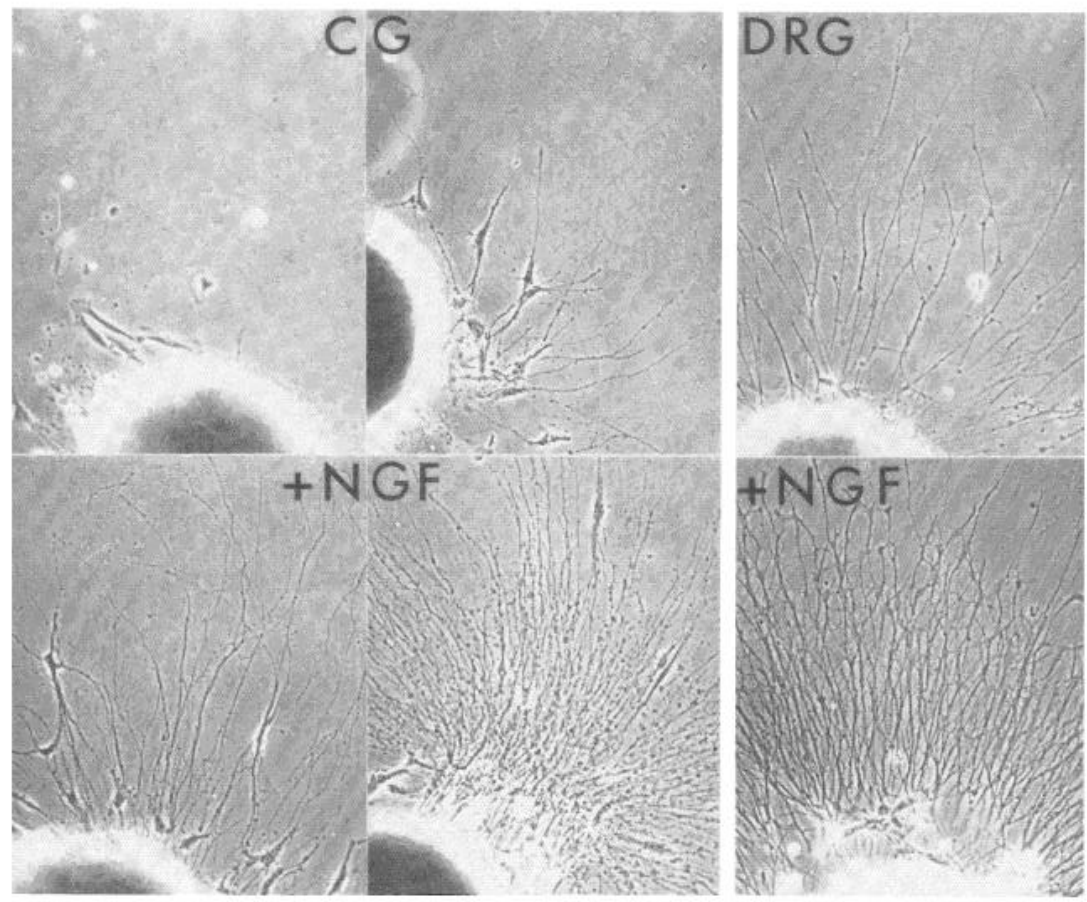

Figure 1. The response of ciliary and dorsal root ganglia to NGF. Phasecontrast micrographs were taken of E.8 ciliary $(C G)$ or dorsal root $(D R G)$ ganglia that had been cultured for $24 \mathrm{hr}$ in medium only (upper row of photographs) or in medium containing $5 \mathrm{ng} / \mathrm{ml}$ of NGF from Collaborative Research (lower row of photographs) on polyornithine-coated dishes pretreated with conditioned medium. There was typically some variability in the density of outgrowth among ciliary ganglia in the same culture. Therefore, two photographs are shown of the control and two of the NGF-treated ciliary ganglion cultures to illustrate the upper and lower range of the observed outgrowth. 


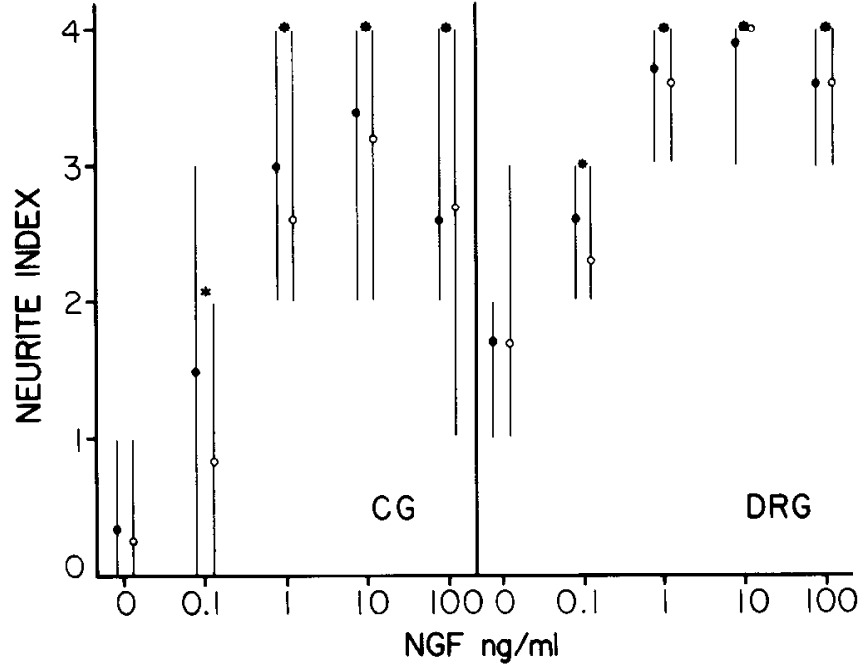

Figure 2. Comparison of the dose response curves to NGF of ciliary and dorsal root ganglia. E.9 ciliary $(C G)$ or dorsal root $(D R G)$ ganglia were plated onto polyornithine-coated dishes in culture medium containing $0,0.1,1,10$, or $100 \mathrm{ng} / \mathrm{ml}$ of NGF. After $24 \mathrm{hr}$, explants were scored for the density and length of neurite outgrowth (neurite index, 0 to 4 scale). Each point represents the mean and range of the observed values. The results of two separate experiments are presented, with the individual points displaced to either side of the actual NGF concentration for clarity. The open circle represents NGF obtained from Dr. Frazier; the solid circle represents NGF obtained from The Research Foundation of the State University of New York. The statistical significance of the difference between the values observed for each concentration of NGF and the control without NGF was determined using the MannWhitney $U$ test on the combined data from the two experiments: $C G$-thin asterisk above 0.1 value, $p<0.01 ; D R G-$ asterisk above 0.1 value, $p<0.002$; thick asterisks above 1,10 , and 100 values for both $C G$ and $D R G, p<0.001$.

than those required to stimulate neurite outgrowth significantly (Fig. 3). Neurites were visibly degenerating even at $24 \mathrm{hr}$ in the ciliary ganglion cultures, as indicated by the presence of ballooned regions of vacant-appearing cytoplasm along the length of many of the neurites. By $48 \mathrm{hr}$, most of the neurites had apparently lysed without leaving visible remnants (Fig. 3). The loss of neurites with time was presumably due to the failure of NGF to prolong the survival of ciliary ganglion nerve cells, as previously shown (Collins and Dawson, 1983).

Neurites extended by dorsal root ganglia in the absence of NGF continued to survive in its absence for the period of observation (Fig. 3). However, the additional neurites extended from dorsal root ganglia upon addition of NGF required this agent for continued survival, since withdrawal of NGF, as previously documented extensively by others, led to a substantial loss of neurites within 24 to $48 \mathrm{hr}$ (data not shown). NGF at a concentration of 0.1 to $1 \mathrm{ng} / \mathrm{ml}$ sufficed to maintain these additional neurites (Fig. 3).

Specificity. It is possible that the culture conditions used in these experiments and/or the nature of the NGF preparations produced a nonspecific stimulation of neurite growth. To test this possibility, two other readily obtained embryonic chick neural explants, from spinal cord and neural retina, were tested for their response to NGF in the same culture conditions used with ciliary and dorsal root ganglia. Both types of explant in control medium exhibited sparse to moderate neuritic growth, but neither the mean density or length of the neurites was significantly increased by NGF in concentrations from 0.1 to $100 \mathrm{ng} / \mathrm{ml}$ (Fig. 4 and Table II). This result suggests that there is some degree of neural specificity in the response to NGF in the present culture conditions. It should be emphasized that this limited result does not demonstrate that neurons in the spinal cord and neural retina of chicken embryos are insensitive to NGF.

To determine the chemical specificity of the effect of NGF on neurite outgrowth in ciliary ganglia, its effects were compared to those of the following compounds: cytochrome $c$ (a protein of similar size and basicity), cpidermal growth factor (the other major growth factor in the mouse submaxillary gland), and insulin (a protein of similar amino acid sequence to NGF). Of these compounds, only insulin had a significant effect on ciliary ganglion neurite outgrowth, although the magnitude of this effect approached that of NGF only at concentrations of insulin 1,000- to 100,000-fold higher (Fig. 5).

Purity. Since there might be some question concerning the purity of commercial NGF preparations, a portion of the Collaborative Research NGF used in these experiments was iodinated and run on an SDS polyacrylamide slab gel under reducing conditions (Fig. 6). Quantitative density tracing of the autoradiogram made from the gel indicated that greater than $98 \%$ of the radioactivity was present as a single major band of appropriate molecular weight for the reduced form of $\beta$-NGF (Fig. 6). Also, greater than $94 \%$ of the radioactivity in this preparation could be pelleted using anti-NGF antibodies (Table III). NGF from the Research Laboratory of the State University of New York was also iodinated and analyzed as above. The results were comparable to those just reported. Greater than $97 \%$ of the radioactivity was present in the major band on SDS polyacrylamide gels, and greater than $90 \%$ of this radioactivity could be precipitated using anti-NGF antibody.

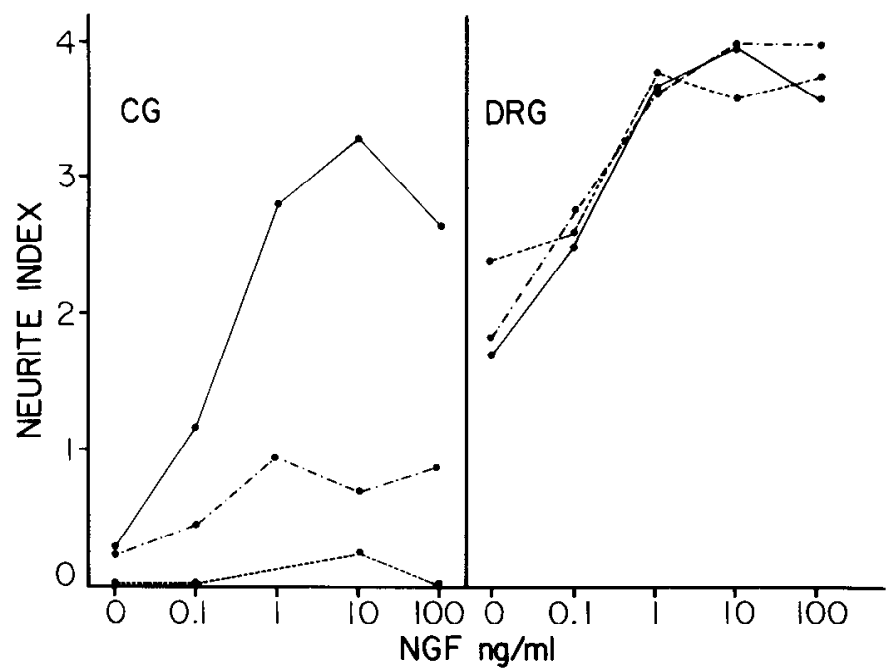

Figure 3. The effect of NGF on the survival of neurites extended from ciliary and dorsal root ganglia. The neurite index ( 0 to 4 scale) of the cultures described in the legend to Figure 2 was recorded at $1(-), 2(\cdot-\cdot)$, and $4(--)$ days after plating. The values given are the means of the combined data from the two experiments. 


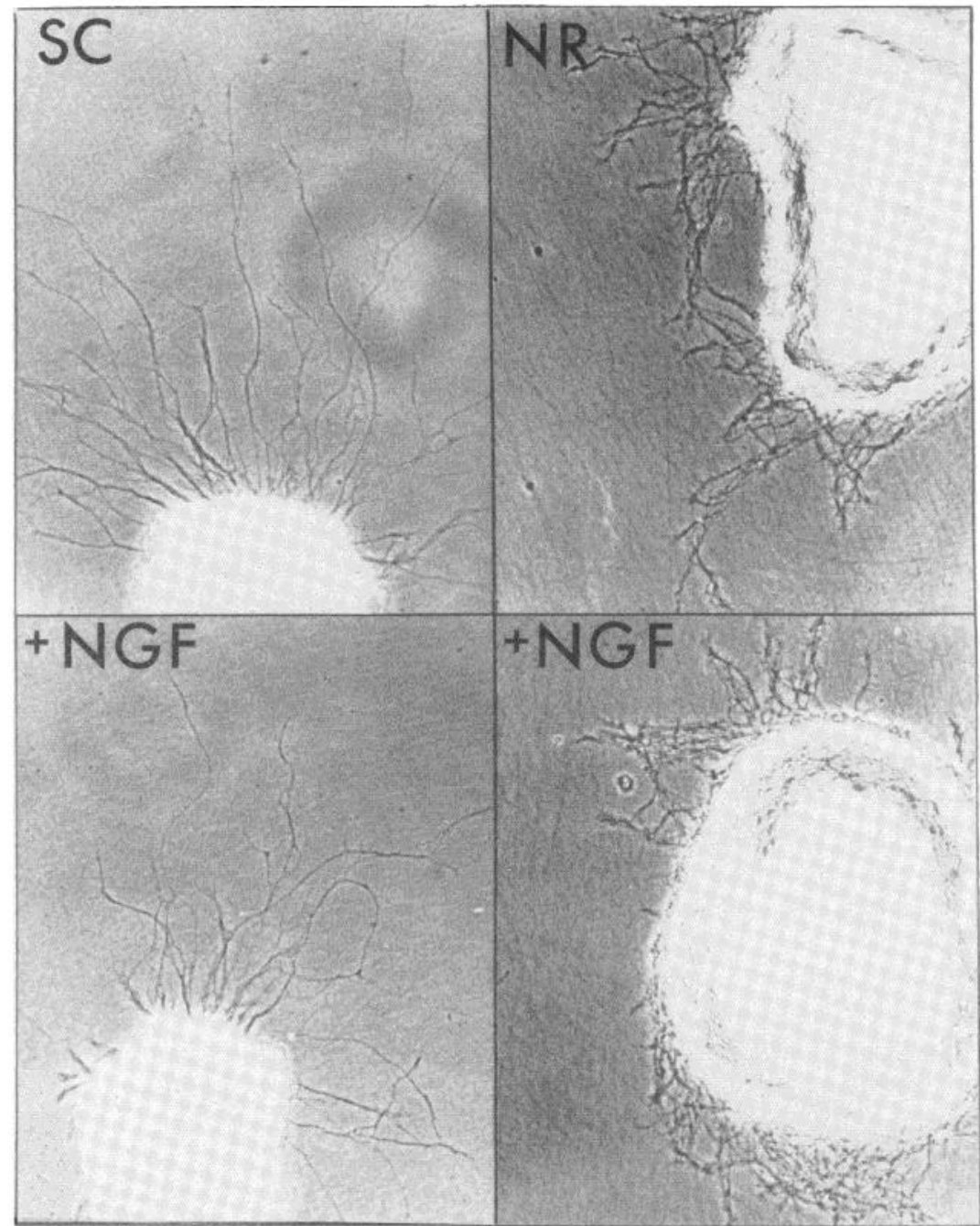

Figure 4. The response of spinal cord and neural retina explants to NGF. Phase-contrast micrographs were taken of explants of E.6 spinal cord (SC) or neural retina $(N R)$ that had been cultured for $24 \mathrm{hr}$ in medium only (upper row of photographs) or in medium containing $25 \mathrm{ng} / \mathrm{ml}$ of NGF from Collaborative Research (lower row of photographs) on polyornithinecoated dishes pretreated with conditioned medium. The photographs were taken of explants selected at random from among the 12 pieces per culture.

\section{Discussion}

The principal findings of this paper are: (1) Undissociated ciliary ganglia, as well as dissociated neurons freed of non-neuronal cells, respond to mouse submaxillary gland NGF with increased neurite growth. The magnitude of this response is less than the response of dorsal root ganglia, but it occurs at the same low concentrations of NGF. (2) NGF does not promote the survival of ciliary ganglion nerve cells, even at concentrations much higher than those that significantly stimulate neurite growth. (3) There is neuronal specificity of neurite stimulation in our experimental conditions, since NGF does not increase outgrowth from explants of spinal cord and neural retina. (4) The stimulation of ciliary ganglion neurite growth by NGF does not require the substrateassociated material from conditioned medium. (6) A number of control experiments and direct assessments of purity significantly decrease the likelihood that the effect on neurite growth is due to a contaminant rather than to NGF.

Undissociated ganglia. Ganglionic explants represent a more intact embryonic system than do dissociated neuronal cultures freed of non-neuronal cells. The latter can be used to demonstrate a direct action of putative trophic agents on nerve cells. However, the trophic effects observed using dissociated neurons will not necessarily apply to the more embryonically relevant intact system. In intact ganglia the high density of non-neuronal cells may supply a similar-acting trophic agent, thus rendering nil the effects of exogenously supplied material. Also, the dissociation process may render neurons responsive to external influences which would have little or no effect in a more normal situation. We recently observed that dissociated neurons from the chick embryo ciliary ganglion quite unexpectedly exhibit a response to NGF (Collins and Dawson, 1983). For the reasons discussed above it was important to examine the effects of NGF 


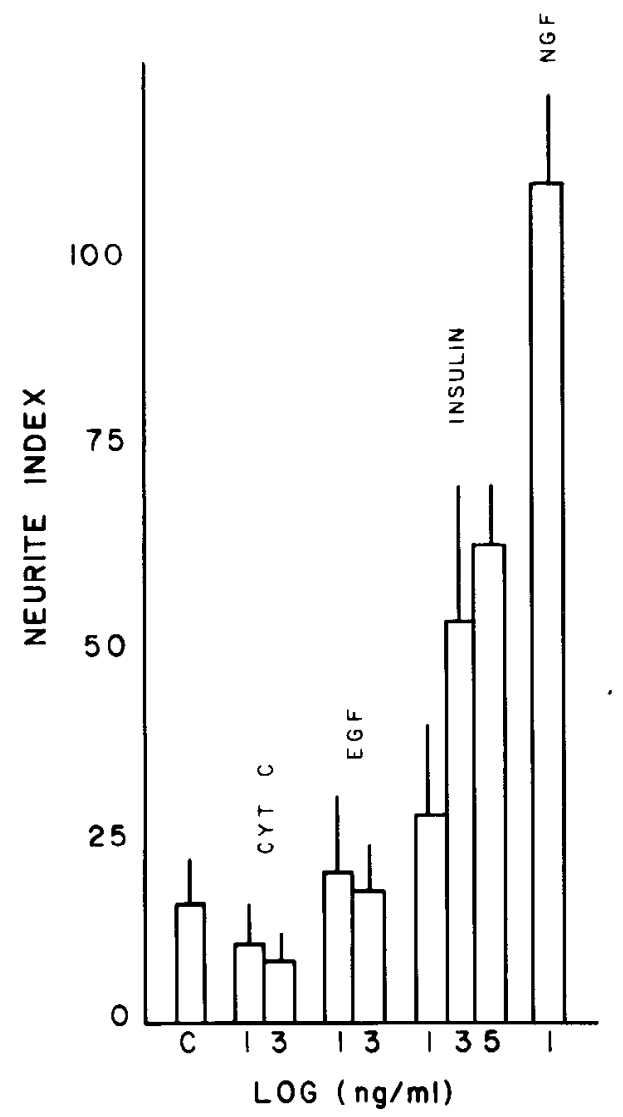

Figure 5. A comparison of the effects of various agents on the extent of neurite outgrowth from ciliary ganglia. E.8 ciliary ganglia were plated onto polyornithine-coated dishes in culture medium containing $10^{1}, 10^{3}$, or $10^{5}$ (insulin only) $\mathrm{ng} / \mathrm{ml}$ of cytochrome $c(C Y T C)$, epidermal growth factor $(E G F)$, bovine insulin, or NGF (Collaborative Research). After $24 \mathrm{hr}$, the neurite index (reticle assay) was measured. The values presented are the mean and range for duplicate experiments.

on undissociated ciliary ganglia as a first step in following up the potential biological significance of our observation on dissociated neurons.

The present results indicate that four different, purified preparations of mouse submaxillary gland NGF caused a significant increase in the density and length of neurites extended from the parasympathetic ciliary ganglion of chicken embryos in vitro. The concentrations of NGF required to elicit this response were similar to those required by dorsal root ganglia (Fig. 2). Detectable increases occurred at concentrations of NGF as low as 0.1 $\mathrm{ng} / \mathrm{ml}\left(4 \times 10^{-12} \mathrm{M}\right)$. This effect of NGF on intact ciliary ganglia is analogous to its effect on isolated ciliary ganglion neurons, where similarly low concentrations of NGF caused a 2-fold increase in the rate of neurite elongation in dissociated ciliary ganglion cultures freed of non-neuronal cells (Collins and Dawson, 1983). Presumably, therefore, the effect of NGF on undissociated ganglia is due to a direct effect on the ganglionic nerve cells.

The present results with intact ganglia exclude the possibility from our previous study that dissociation is necessary for ciliary ganglion neurons to become respon- sive to NGF. This eliminates a number of possible artifactual causes of the NGF response - for example, trypsin modification of neuronal surface receptors. While dissociation is not required, it remains to be determined whether removal from the embryonic environment is a necessary condition or if, in fact, ciliary ganglia are also responsive to NGF in vivo.

Chemical and neuronal specificity. Neurite growth from explants of chick embryo spinal cord and neural retina is not measurably affected by NGF in the same condition in which the ciliary ganglion response is manifested. 'This suggests that we have not simply created culture conditions in which the NGF preparations act as a nonspecific stimulus to neurite growth.

One possibility which can never be completely excluded is that the effect of preparations of NGF on the ciliary ganglion is due to a contaminant. The following evidence is relevant to this possibility: (1) The concentration of NGF required for this response is low and is approximately the same as that required for an analogous effect on sympathetic chain (Collins and Dawson, 1983) or dorsal root ganglion neurons (Fig. 2). (2) All five preparations of NGF so far tested have had the same effect (the present report and Collins and Dawson, 1983). The purity of two of the preparations was assayed by the standard criteria of polyacrylamide gel electrophoresis and immunoprecipitation of iodinated material (Fig. 6, Table III) and was found to be well within the purity typically quoted for the NGF used in receptor studies (e.g., Claude et al., 1982; Rohrer and Barde, 1982). (3) The effect is blocked by affinity-purified antibody to NGF (Collins and Dawson, 1983). The antibody in these studies was raised against a preparation of NGF different from the ones on which it was tested. 'Therefore, if the inhibitory antibody is directed against a contaminant, it is a contaminant common to the immunizing and tested NGF preparations. Also, the effect cannot be accounted for by contamination of the NGF with mouse submaxillary gland epidermal growth factor (Fig. 5) or with renin, resulting in the production of angiotensins (Collins and Dawson, 1983). (4) A molecule with similar physical properties, cytochrome c, had no effect on neurite growth. However, a molecule related in amino acid sequence to NGF, insulin (Bradshaw, 1978), had an effect similar to NGF, but only at much higher concentrations. This evidence favors the interpretation that the effects we have observed on ciliary ganglion neurite growth are due to NGF.

Culture requirements. Our previous study suggested the possibility that the material deposited onto the polyornithine substratum by conditioned medium is necessary for the response of dissociated ciliary ganglion neurons to NGF (Collins and Dawson, 1983). Since the dissociated neurons require conditioned medium pretreatment of the substratum in order to initiate outgrowth (Collins, 1978b), and since NGF will not substitute for this pretreatment (Collins and Dawson, 1983), it was not possible to eliminate conditioned medium trcatment as a predisposing factor in the response. However, the present results clearly indicate that intact ganglia do not require pretreatment of the polyornithine substratum in order to extend neurites in response to 


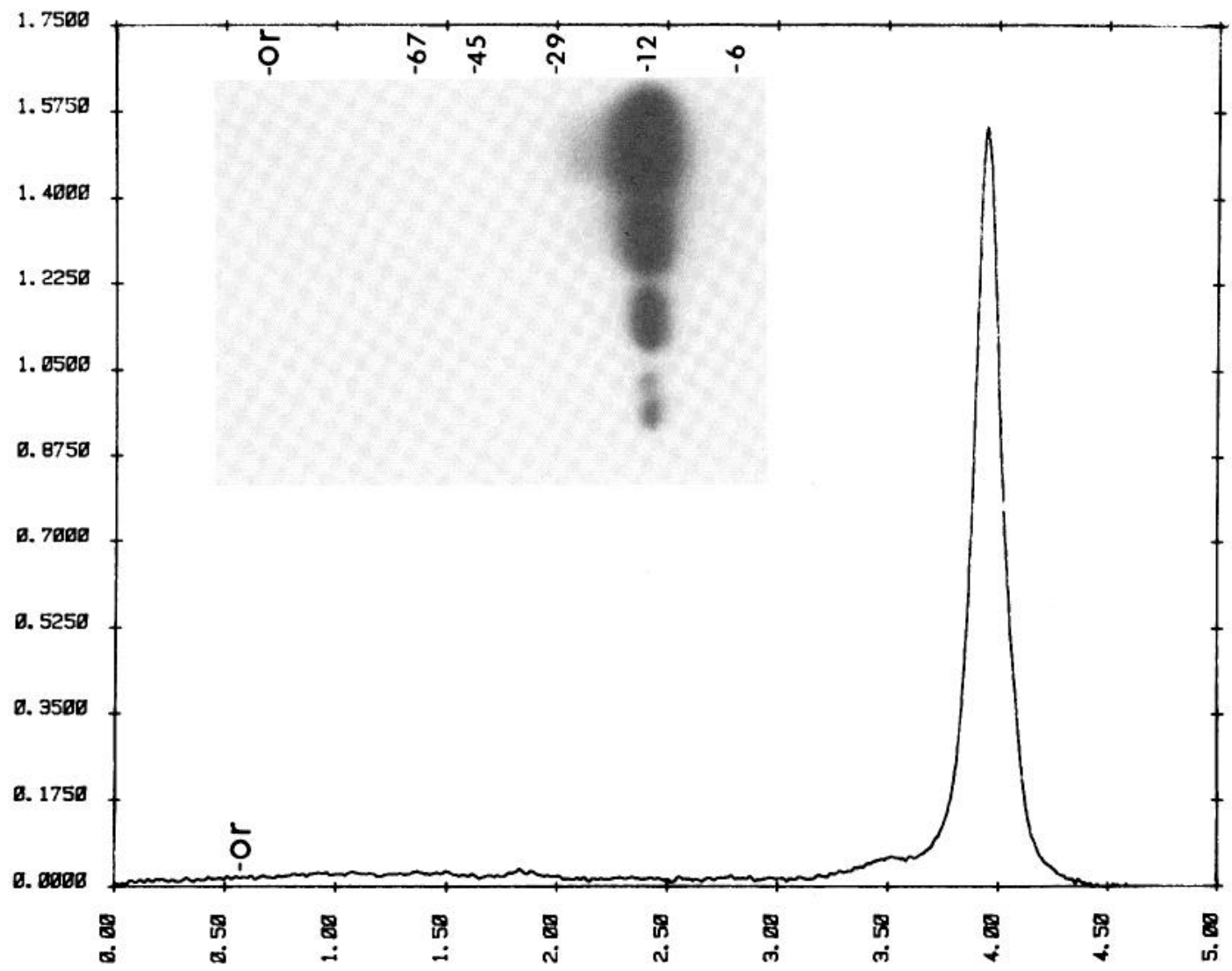

Figure 6. Polyacrylamide gel electrophoresis of iodinated NGF. NGF from Collaborative Research was labeled with ${ }^{125} \mathrm{I}$ and run on an SDS polyacrylamide slab gel under reducing conditions. The inset is a photograph of an autoradiogram of the gel, showing adjacent lanes containing increasing concentrations of the labeled NGF. The position of the origin (or) and of protein molecular weight standards (in kilodaltons) is indicated above the inset. The main figure is an optical density tracing of that lane of the autoradiogram in which the density of the major band was still within the readable range of the spectrophotometer (the second lane from the bottom in the inset).

TABLE III

Binding of [ $\left.^{125} I\right] N G F$ to affinity-purified anti-NGF antibody

Twenty nanograms of $\left[{ }^{125} \mathrm{I}\right] \mathrm{NGF}(12 \mu \mathrm{Ci} / \mathrm{ml})$ were incubated in 0.2 $\mathrm{ml}$ of complete medium $\pm 10 \mu \mathrm{g}$ of affinity-purified anti-NGF antibody for $45 \mathrm{~min}$ on ice. Then $50 \mu \mathrm{l}$ of a suspension of formaldehyde-fixed Staphylococcus aureus bacteria were added to the appropriate tubes for an additional $15 \mathrm{~min}$. The bacteria were pelleted by microfuge, and the radioactivity $(\mathrm{cpm})$ in an aliquot of the supernatant was measured.

\begin{tabular}{ccc}
\hline Anti-NGF & S. aureus & $\mathrm{cpm}$ \\
\hline & $\mu l$ & \\
& & 47,771 \\
$10 \mu \mathrm{g}$ & 50 & 38,788 \\
& 50 & 2,056 \\
\hline
\end{tabular}

NGF. It is possible that the ganglion itself is conditioning the substratum, but it should be noted that by $20 \mathrm{hr}$ in NGF some neurites have extended up to $750 \mu \mathrm{m}$ from the ganglion's perimeter, while few non-neuronal cells have left the ganglion.

Previous negative results. Our results differ from previous reports that NGF fails to affect ciliary ganglion neurons in vitro (Helfand et al., 1976; Ebendal 1979).
This discrepancy is presumably due to specific differences in culture methods (see detailed discussion in Collins and Dawson, 1983). The lack of responsiveness to NGF of dissociated ciliary ganglion neurons, reported by Helfand et al. (1976), is probably due to the lack of pretreatment of the polyornithine substratum with conditioned medium, in the absence of which neurite outgrowth fails to occur even with added NGF (Collins and Dawson, 1983). The negative results of Ebendal (1979) using intact ciliary ganglia are more difficult to explain. One possibility is that since Ebendal assayed neurite growth after $48 \mathrm{hr}$, substantial lysis of the neurites by that time may have obscured a positive result (Fig. 3). It is also possible that the collagen substratum he used may be less supportive of ciliary ganglion neurite growth in the absence of cell-derived factors than is polyornithine.

Neuronal survival. NGF fails to prevent the rapid degeneration of ciliary ganglion neurites, which is visibly beginning by $24 \mathrm{hr}$ (Fig. 3 ). Neurite degeneration is presumably a result of the failure of NGF to maintain survival of the ciliary ganglion nerve cells (Collins and Dawson, 1983). There is no apparent increased survival in these experiments, even when the concentration of 
NGF is increased 1000 -fold over the concentration required to stimulate neurite outgrowth or to promote the survival of dorsal root ganglion neurites. It should be noted that ciliary ganglion neurons can survive in culture for at least several weeks with appropriate additions to the culture medium (Tuttle et al., 1980). Therefore, it seems that the effects of NGF on neurite growth and neuronal survival can be separated. The ciliary ganglion, like PCl2 cells (Greene and Tischler, 1976), may provide a system for studying the mechanism of NGF-promoted neurite outgrowth isolated from effects on cell survival.

Significance. The effect of NGF on neurons of the ciliary ganglion is distinguishable from its extensively documented effect on sympathetic and sensory neurons. In contrast to the action of NGF on the latter, the effect on neurons of the ciliary ganglion is limited to neurite outgrowth, while neuronal survival is apparently unaffected. The magnitude of the effect of NGF on ciliary ganglion neuritic growth is less than with dorsal root ganglia, and there is some variability among ciliary ganglia in their responsiveness to NGF (Figs. 1 and 2). Also, as indicated previously, particular culture conditions may be required for demonstrating the effect of NGF on ciliary ganglia.

It is possible that only a subpopulation of neurons in the ciliary ganglion is responsive to NGF or that the response would be enhanced in ganglia taken at a different developmental stage. However, it should be borne in mind that the response of the ciliary ganglion to NGF may be artificially reduced by the concurrent neuronal cell death noted above. Also, the extent of cell death in individual ciliary ganglia might vary, accounting for some of the observed variability among ganglia in their responsiveness to NGF. Despite this, the NGF-induced increase in ciliary ganglion neurite density and length is significant and, with some ganglia in each culture, can be quite extensive (Fig. 1).

The present results appear to correspond with other recent evidence that specific populations of cholinergic neurons can respond to NGF. Responsive neurons seem to be those which also demonstrate active uptake and retrograde axonal transport of radiolabeled NGF in vivo. Among central neurons, Schwab et al. (1979) reported specific uptake of NGF by cholinergic neurons projecting to the rat hippocampal formation. Gnahn et al. (1983) have now demonstrated that choline acetyltransferase is increased in response to NGF in regions of the rat's brain containing these cholinergic neurons both in vivo and in vitro. In the peripheral nervous system, Max et al. (1978) demonstrated specific uptake and transport of NGF by some neurons in the rat and chick ciliary ganglion. We have now shown that NGF also has a stimulatory effect on fiber outgrowth of these parasympathetic cholinergic neurons in vitro.

The possible role, if any, of NGF or NGF-related agents in the normal development and functioning of these cholinergic neurons remains to be determined. As a step in this direction, we are presently testing the possibility that ciliary ganglion neurons possess surface receptors capable of binding NGF at physiological concentrations.

\section{References}

Bradshaw, R. A. (1978) Nerve growth factor. Annu. Rev. Biochem. 47: 191-216.

Claude, P., E. Hawrot, D. A. Dunis, and R. B. Campenot (1982) Binding, internalization, and retrograde transport of ${ }^{125} \mathrm{I}-$ nerve growth factor in cultured rat sympathetic neurons. J. Neurosci. 2: 431-442.

Collins, F. (1978a) Axon initiation by ciliary neurons in culture. Dev. Biol. 65: 50-57.

Collins, F. (1978b) Induction of neurite outgrowth by a conditioned medium factor bound to the culture substratum. Proc. Natl. Acad. Sci. U. S. A. 75: 5210-5213.

Collins, F., and A. Dawson (1983) An effect of nerve growth factor on dissociated parasympathetic neurons. Proc. Natl. Acad. Sci. U. S. A. 80: 2091-2094.

Collins, F., and M. R. Lee (1982) A reversible developmental change in the ability of ciliary ganglion neurons to extend neurites in culture. J. Neurosci. 2: 424-430.

Ebendal, T. (1979) Stage dependent stimulation of neurite outgrowth exerted by nerve growth factor and chick heart in cultured embryonic ganglia. Dev. Biol. 72: 276-290.

Gnahn, H., F. Hefti, R. Heumann, M. E. Schwab, and H. Thoenen (1983) NGF-mediated increase of choline acetyltransferase (ChA'I) in the neonatal rat forebrain: Evidence for a physiological role of NGF in the brain? Brain Res. 285: 45-52.

Greene, L. A., and A. Tischler (1976) Establishment of noradrenergic clonal line of rat adrenal pheochromocytoma cells which respond to nerve growth factor. Proc. Natl. Acad. Sci. U. S. A. 73: 2424-2428.

Greene, I. A., and E. M. Shooter (1980) The nerve growth factor: Biochemistry, synthesis, and mechanism of action. Annu. Rev. Neurosci. 3: 353-402.

Harper, G. P., and H. Thoenen (1980) Nerve growth factor: Biological significance, measurement, and distribution. J. Neurochem. 34: 5-16.

Helfand, S. L., G. A. Smith, and N. K. Wessells (1976) Survival and development in culture of dissociated parasympathetic neurons from ciliary ganglia. Dev. Biol. 50: 541-547.

Honegger, $\mathrm{F}$., and D. Lenoir (1982) Nerve growth factor (NGF) stimulation of cholinergic telencephalic neurons in aggregating cell cultures. Dev. Brain Res. 3: 229-238.

Laemmli, U. K. (1970) Cleavage of structural proteins during the assembly of the head of bacteriophage T4. Nature 227 : 680-685.

Lehman, J. M., W. C. Speers, D. E. Swartzendruber, and G. B. Pierce (1974) Neoplastic differentiation: Characteristics of cell lines derived from murine teratocarcinoma. J. Cell Physiol. 84: 13-28.

Max, S. R., M. Schwab, M. Dumas, and H. Thoenen (1978) Retrograde axonal transport of nerve growth factor in the ciliary ganglion of the chick and the rat. Brain Res. 159: 411415.

Rohrer, H., and Y. $-\Lambda$. Barde (1982) Presence and disappearance of nerve growth factor receptors on sensory neurons in culture. Dev. Biol. 89: 309-315.

Schwab, M. E., U. Otten, Y. Agid, and H. Thoenen (1979) Nerve growth factor (NGF) in the rat CNS: Absence of specific retrograde axonal transport and tyrosine hydroxylase induction in locus coeruleus and substantia nigra. Brain Res. 168: 473-483.

Studier, F. W. (1973) Analysis of bacteriophage 17 early RNAs and proteins on slab gels. J. Mol. Biol. 79: 237-248.

Tuttle, J. B., J. B. Suszkiw, and M. Ard (1980) Long term survival and development of dissociated parasympathetic neurons in culture. Brain Res. 183: 161-180. 